\title{
Bio-bibliografia ragionata di Isabella Andorlini
}

\author{
Luca Iori / Margherita Centenari
}

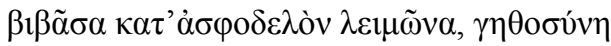

Hom. Od. XI 539-540

Isabella Andorlini nasce a Firenze il 28 aprile 1955. Dopo aver conseguito la laurea in Lettere Classiche all'Università di Firenze (1979), approfondisce lo studio della papirologia greca e nel 1984 ottiene presso l'ateneo fiorentino la specializzazione in Papirologia e Paleografia. Tra 1979 e 1984, partecipa regolarmente alla Missione Archeologica Italiana in Egitto, prestando servizio nelle campagne di scavo ad Antinoupolis organizzate dall'Istituto Papirologico "Girolamo Vitelli", sotto la guida di Manfredo Manfredi. Negli anni successivi frequenta l'Istituto di Filologia Classica dell'Università di Urbino, dove nel 1986 discute la propria tesi di dottorato in Letteratura Greca dedicata a un importante trattato di medicina su papiro, di cui riunisce ed edita i frammenti dispersi tra le collezioni di Berkeley, Copenhagen, Firenze, Lund e Milano (supervisore: Bruno Gentili; il testo sarà poi pubblicato nel 1995 sotto gli auspici dell'Istituto Vitelli). Durante questo percorso, Isabella Andorlini acquisisce una formazione filologica e papirologica di rara solidità, che le permette di unire a una raffinata sensibilità per la lingua e la letteratura greca, una sicura padronanza della tecnica ecdotica e notevoli competenze nello scavo e nel restauro dei documenti papiracei. Da subito, il suo lavoro di ricerca offre brillanti risultati nel campo della papirologia medica, ambito nel quale si specializza, pubblicando, tra gli altri, rilevanti contributi sul "Bulletin of the American Society of Papyrologists" e su "Yale Classical Studies", e intervenendo al XVII e al XIX Congresso Internazionale di Papirologia (Napoli 1983; Il Cairo 1989). L'interesse per la medicina antica si accompagna però, fin dagli inizi, a uno scrupoloso apprendistato filologico, che la porta ad editare testi letterari e documentari all'interno di prestigiose raccolte: Oxyrhynchus Papyri (vol. XLIX), Rendel Harris Papyri (vol. II), Corpus dei Papiri Filosofici Greci e Latini (vol. I.1*).

Dopo alcuni anni di insegnamento nelle scuole secondarie, nel 1992 Isabella Andorlini diventa Ricercatrice presso l'Istituto Papirologico "G. Vitelli" di Firenze, dove lavora fino al 2005. In questo periodo estende e approfondisce le 
proprie ricerche di tema medico, avviando una produzione scientifica ricca e originale, che le consente di affermarsi in breve tempo come specialista di fama internazionale. Dopo aver curato l'ampia sezione dedicata a L'apporto dei papiri alla conoscenza della scienza medica antica all'interno dell'opera enciclopedica Aufstieg und Niedergang der römischen Welt (ANRW, 1993), vara l'ambizioso progetto Corpus dei Papiri Greci di Medicina, che si propone di raccogliere, rieditare e commentare tutti $\mathrm{i}$ papiri di contenuto medico già pubblicati, insieme a quelli che sarebbero stati resi noti in corso d'opera. Presentato alla comunità papirologica durante il XXI Congresso Internazionale di Berlino (1995), il Corpus si arricchì in pochi anni di quattro importanti volumi, che ospitavano $\mathrm{i}$ contributi dei maggiori studiosi del settore: Specimina per il Corpus dei Papiri Greci di Medicina (1997), Estratto provvisorio dal Corpus dei Papiri Greci di Medicina (1998), Greek Medical Papyri I (2001), Testi medici su papiro (2004). Nel quadro di questa iniziativa, edita tra 1995 e 2005 numerosi testi, affiancandovi un'introduzione manualistica alla medicina antica scritta insieme ad Arnaldo Marcone: Medicina, medico e società nel mondo antico (2004).

Negli anni trascorsi all'Istituto Vitelli, Isabella Andorlini pubblica anche i suoi primi saggi sulla società e la cultura letteraria dell'Egitto antico, sulla storia della Papirologia (con particolare riferimento agli scavi di Antinoe e alle collezioni fiorentine e dell'Università di Lund) e sulle tecnologie digitali applicate alla ricerca papirologica. Prosegue inoltre la sua attività di editrice di papiri letterari (Eschine, Isocrate, Omero, Platone, Plutarco, i Pitagorici) e documentari. Tra questi ultimi spicca il lavoro, condotto a quattro mani con Klaus Maresch, sui frammenti dell'archivio di Aurelius Ammon, avvocato panopolitano vissuto nel IV sec. d.C. (P. Ammon II = Pap.Col. XXVI/2 [2006]). Nello stesso periodo, matura e consolida la sua attitudine alla ricerca internazionale, trascorrendo vari periodi all'estero con il sostegno di prestigiose fellowships: nel 1987 e nel 1997 è ospite del Wellcome Institute for the History of Medicine di Londra, nel 1997 della Fondation Hardt di Ginevra, nel 2002 dell'Institute for Advanced Study di Princeton (Historical Studies), mentre tra 1994 e 2006 è più volte visiting scholar presso l'Universität zu Köln per preparare l'edizione dei P. Ammon. Nel 1998, insieme a Manfredo Manfredi, Guido Bastianini e Giovanna Menci, organizza il XXII Congresso Internazionale di Papirologia, tenutosi a Firenze.

Come meritato riconoscimento della sua brillante carriera, nel 2005 Isabella Andorlini ottiene la cattedra di Papirologia presso l'Università di Parma, dove è chiamata a insegnare come Professore Associato e dove presterà servizio fino al 2016, conseguendo nel 2013 l'idoneità a Professore di Prima Fascia. A Parma, oltre a dedicarsi a un'intensa e generosa attività didattica, prosegue il suo impegno di ricerca lungo le linee già impostate, approfondendole e ampliandole in molteplici direzioni. In campo medico, il consueto impegno sul fronte ecdotico si concretizza nell'edizione commentata di una ventina di papiri, tra cui 
spiccano due importanti testi di età ellenistica (P.ÄkNo. 1 e 2), pubblicati nel 2016 insieme a Robert Daniel nella serie Papyrologica Coloniensia (XXXVIII). I suoi interessi intersecano inoltre, sempre più spesso, temi e problemi connessi alla dimensione sociale, culturale e materiale della medicina antica. I contributi, spesso anticipati in convegni internazionali (Ann Arbor, Freudenstadt, British Academy of London, Ginevra, Heidelberg - dove fu visiting scholar nel 2013 -, Parigi, New York, Berlino), approfondiscono così, con rinnovata originalità, le modalità di redazione e trasmissione dei testi medici, la storia delle malattie e delle pratiche di cura, le strutture assistenziali e la contaminazione tra scienza greca e medicina egizia in epoca ellenistico-romana.

Negli anni parmigiani si consolida infine la sua spiccata vocazione alla progettualità, declinata su scala nazionale e internazionale. Oltre a completare l'allestimento di un altro volume del Corpus dei Papiri Greci di Medicina (Greek Medical Papyri II, 2009) e ad avviare il progetto di un terzo, dà vita a una ricca serie di iniziative scientifiche in cui coinvolge stabilmente un piccolo gruppo di giovani collaboratori reclutati tra gli studenti dei corsi di Papirologia. A partire dal 2006, attiva una convenzione con il Center for the Tebtunis Papyri (Bancroft Library, University of California, Berkeley) per l'edizione di papiri tolemaici e romani, che produce un vivace seminario permanente ("Papiri inediti da Tebtynis") e una serie di giornate di studio ad esso collegate, che, accompagnate da un ricco calendario di conferenze, attirano ogni anno a Parma insigni antichisti e papirologi (Guido Bastianini, Tiziano Dorandi, Jean-Luc Fournet, Nikolaos Gonis, Ann Hanson, Sabine Huebner, Todd Hickey, David Leith, John Lundon, Caroline Magdelaine, Anastasia Maravela, Vivian Nutton). Attraverso la proficua collaborazione con la cattedra di Archeologia e Storia dell'Arte Greca e Romana, allora ricoperta da Sara Santoro, partecipa inoltre a un progetto PRIN 2007 ("Atelier, fabbrica e cantiere. Tecniche e cultura della produzione nel mondo romano"), di cui diviene coordinatrice dopo la chiamata della collega all'Università di Chieti, promuovendo contestualmente attività di taglio divulgativo presso il Museo Archeologico Nazionale di Parma. Infine, a partire dal 2010, approfondisce le potenzialità offerte dalle risorse informatiche applicate all'edizione e al commento dei testi papiracei. In quell'anno entra a far parte del collegio dei Senior Editors della piattaforma Papyri.info ( $<$ http://www.papyri.info $>$ ) ed elabora, nel corso del 2011/2012, un progettopilota di digitalizzazione dell'intera letteratura medica greca su papiro ( $C P G M$ Online), che ottiene un prestigioso e ingente finanziamento da parte dello European Research Council (ERC Advanced Grant 2013).

Il progetto ("Online Humanities Scholarship: A Digital Medical Library Based on Ancient Texts", DIGMEDTEXT), pensato come naturale complemento del Corpus dei Papiri Greci di Medicina, rappresenta il degno coronamento della carriera scientifica di Isabella Andorlini. Grazie al suo impegno e al suo indirizzo programmatico, il progetto produce, nello spazio di pochi anni, una pionieristica 
banca dati testuale comprensiva di oltre trecento testi medici - ora tutti disponibili all'interno del Digital Corpus of Literary Papyrology ( $<\mathrm{http}$ ://www.litpap.info $>$ ) e avvia parallelamente un database lessicale dedicato alla terminologia medica (Medicalia Online, <http://www.medicaliaonline.unipr.it $>$ ). Inoltre, com'era nei desideri di Isabella, per tre anni Parma diviene il centro di una fitta rete di scambi internazionali, che consentono a studenti, dottorandi e studiosi provenienti da tutto il mondo di collaborare ai lavori di digitalizzazione, impegnandosi in workshops, training sessions e in una serie di seminari e convegni, culminati nella conferenza finale tenutasi nel novembre 2016, pochi giorni prima della sua prematura scomparsa. Resta il profondo e doloroso rammarico che Isabella non abbia potuto vedere compiutamente realizzati i frutti del suo progetto e che la malattia l'abbia colpita proprio nel momento più alto e maturo della sua carriera, ma gli importanti risultati conseguiti, anche per opera dei suoi collaboratori, dimostrano oggi, più di ogni altra testimonianza, l'eccellenza della sua ricerca e delle sue doti di insegnante.

\section{Bibliografia di Isabella Andorlini}

\section{Papirologia medica}

\section{Il progetto del Corpus dei Papiri Greci di Medicina}

1997a. 'Progetto per il Corpus dei Papiri Greci di Medicina', in Akten des 21. Internationalen Papyrologenkongresses (Berlin, 13-19 8. 1995), hrsg. von B. Kramer, W. Luppe, H. Maehler, G. Poethke, vol. I, Stuttgart - Leipzig 1997, pp. 17-24.

1997b. (a c. di). 'Specimina' per il Corpus dei Papiri Greci di Medicina. Atti dell'incontro di studio (Firenze, 28-29 marzo 1996), Firenze 1997.

1998. (a c. di). Estratto provvisorio dal Corpus dei Papiri Greci di Medicina. Fascicolo presentato in occasione del XXXII Congresso Internazionale di Papirologia (Firenze, 23-25 agosto 1998), Firenze 1998.

2001. (a c. di). Greek Medical Papyri, vol. I, Firenze 2001.

2004. (a c. di). Testi medici su papiro. Atti del Seminario di studio (Firenze, 3-4 giugno 2002), Firenze 2004. 2009. (a c. di). Greek Medical Papyri, vol. II, Firenze 2009.

2017. 'Il corpus dei papiri medici online: la piattaforma editoriale', in Atti del VII colloquio internazionale sull'ecdotica dei testi medici greci (Procida, 11-13 giugno 2013), a c. di A. Roselli, Napoli 2018 (in c.d.s.).

\section{Edizione, ricostruzione e commento di testi medici}

1981a. 'Ricette mediche nei papiri. Note d'Interpretazione e Analisi di Ingredienti ( $\sigma \mu \hat{\rho} \rho v \alpha$,

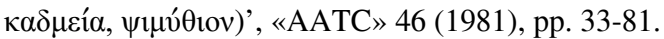

1981b. 'P. Grenf. I 52: note farmacologiche', «BASP» 18 (1981), pp. 1-25.

1981c. 'Una ricetta del medico Cassio: P. Harris 46', «BASP» 18 (1981), pp. 97-100.

1983. 'Ricetta per un $\mu \alpha \lambda_{\alpha} \alpha \mu \alpha$ ', in Trenta testi greci da papiri letterari e documentari editi in occasione del XVII Congresso Internazionale di Papirologia (Napoli, 19-26 maggio 1983), a c. di M. Manfredi, Firenze 1983, pp. 64-68 (nr. 19). 
1985. 'Istruzioni dietetiche e farmacologiche', «YCS» 28 (1985), pp. 49-56.

1991. 'Una trattazione "Sui veleni e sugli antidoti” (PL 68)', «AnPap» 3 (1991), pp. 85-101.

1992a. 'Papiri e medicina: POxy II 234 + POxy LII 3654', in Proceedings of the XIXth International Congress of Papyrology (Cairo, 2-9 September 1989), ed. by A.H.S. ElMosalamy, vol. I, Cairo 1992, pp. 375-390.

1992b. 'Prescrizione medica', in Dai Papiri della Società Italiana. Omaggio al XX Congresso Internazionale di Papirologia (Copenhagen, 23-29 agosto 1992), Firenze 1992, pp. 24-28 (nr. 5).

1993. 'Riesame di PVindob. G 29368. Sulle 'parotidi', in Studi di lessicologia medica antica («Opuscula Philologa» 6), a c. di S. Boscherini, Bologna 1993, pp. 7-29.

1995a. Trattato di medicina su papiro, Firenze 1995.

1995b. 'Ricettario medico', in Dai Papiri della Società Italiana. Omaggio al XXI Congresso Internazionale di Papirologia (Berlino, 13-19 agosto 1995), Firenze 1995, pp. 10-25 (nr. 3).

1996. 'Il Papiro di Strasburgo inv. G 90 e l'oftalmologia di Aezio', in Storia e ecdotica dei testi medici greci. Atti del II Convegno Internazionale (Parigi, 24-26 maggio 1994), a c. di A. Garzya, Napoli 1996, pp. 7-30.

1997. 'Trattato o catechismo? La tecnica della flebotomia in PSI inv. CNR 85/86', in 'Specimina' per il Corpus dei Papiri Greci di Medicina, cit., pp. 153-168.

1998. 'MP' 2352. Hippocrates, De fracturis 37', in Estratto provvisorio dal Corpus dei Papiri Greci di Medicina, cit., pp. 1-3.

1999. 'Testi medici per la scuola: raccolte di definizioni e questionari nei papiri', in I testi medici greci. Tradizione e ecdotica. Atti del III Convegno Internazionale (Napoli, 15-18 ottobre 1997), a c. di A. Garzya, J. Jouanna, Napoli 1999, pp. 7-15.

2001a. 'Hippocrates, De fracturis 37', Greek Medical Papyri, vol. I, cit., pp. 3-8 (nr. 1).

2001b. 'Medical Prescriptions', ibidem, pp. 109-118 (nr. 10).

2001c. 'Prescriptions for Plasters', ibidem, pp. 119-129 (nr. 11).

2001d. 'Medical Recipe for a Compress', ibidem, pp. 131-138 (nr. 12).

2003. 'Un anonimo del genere degli Iatromathematikà', in Trasmissione e ecdotica dei testi medici greci. Atti del IV Convegno Internazionale (Parigi, 17-19 maggio 2001), a c. di A. Garzya, J. Jouanna, Napoli 2003, pp. 7-23.

2004. 'Un ricettario da Tebtynis: parti inedite di PSI 1180', in Testi medici su papiro, cit., pp. 81-118. 2005. 'Note di lettura ed interpretazione a PSI IV 299: un caso di tracoma', in Scrivere Leggere Interpretare. Studi di Antichità in onore di Sergio Daris, a c. di F. Crevatin, G. Tedeschi, Trieste 2005, pp. 7-12.

2006. 'Frammento di una trattazione De ossibus: rilettura di PUG II 51 (sec. I d.C.)', in Ecdotica e ricezione dei testi medici greci. Atti del V convegno internazionale (Napoli, 1-2 ottobre 2004), a c. di V. Boudon-Millot, J. Jouanna, A. Garzya, A. Roselli, Napoli 2006, pp. 83-91.

2007. 'Riconsiderazione di PSI II 117: Sorani Gynaecia', in La science médicale antique: nouveaux regards. Études réunies en l'honneur de Jacques Jouanna, éd. par V. Boudon-Millot, A. Guardasole, C. Magdelaine, Paris 2007, pp. 41-71.

2008a. 'Hippocrates, Aph. III 20, 23-24 (cum sch. ad III 24 et 27); III 29, 31; IV 1 (cum sch.); IV 5 (cum sch. ad IV 4-5)', in Corpus dei Papiri Filosofici Greci e Latini, vol. I.2*, Firenze 2008, pp. 89-96.

2008b. 'Hippocrates, Fract. 37', ibidem, pp. 111-112.

2008c. 'Hippocrates, 36T', ibidem, p. 228.

2008d. (con A. Roselli). 'Hippocrates, Epistulae 3; 4; 4a; 5; 6a', ibidem, pp. 150-157.

2008e. (con A. Roselli). 'Hippocrates, Ep. 3; 4a; 5a; 5; 11', ibidem, pp. 157-162.

2008f. (con A. Roselli). 'Hippocrates, Ep. 4a; 5a; 11', ibidem, pp. 162-167.

2008g. 'Old and New Greek Papyri from Tebtunis in the Bancroft Library of Berkeley: Work in Progress', in Graeco-Roman Fayum - Texts and Archaeology. Proceedings of the Third International Fayum Symposion (Freudenstadt, May 29-June 1, 2007), ed. by S. Lippert, M. 
Schentuleit, Wiesbaden 2008, pp. 1-9.

2009a. 'Medical Treatise (addenda to PSI inv. 3054)', Greek Medical Papyri, vol. II, cit., pp. 3-14 (nr. 1).

2009b. 'Medical Treatise On Prognosis', ibidem, pp. 15-33 (nr. 2).

2009c. 'POxy LIV 3724 revised: arteriace', ibidem, pp. 175-182 (nr. 12).

2010. 'La ricetta medica dell'Anonimo Londinese (P.Brit.Libr. inv. 137v = Suppl. Arist. III 1, p. 76

Diels)', «Galenos» 4 (2010), pp. 39-45.

2012a. 'Ricette aromatiche', in Literarische Texte der Berliner Papyrussammlung. Zur Wiedereröffnung des Neuen Museums (BKT X), hrsg. von F. Reiter, Berlin - Boston 2012, pp. 215-231 (nr. 25).

2012b. 'Frammento medico con elenco di sintomi', in Papyrological Texts in Honor of Roger S.

Bagnall, ed. by R. Ast, H. Cuvigny, T.M. Hickey, J. Lougovaya, Durham (NC) 2012, pp. 1-8 (nr. 1).

2012c. 'Testo medico-terapeutico in P.Erl. 12', «ZPE» 182 (2012), pp. 134-136.

2014. 'Ippocratismo e medicina ellenistica in un trattato medico su papiro', in Hippocrate et les hippocratismes: médicine, religion, société. Actes du XIVe Colloque International Hippocratique (Paris, 8-10 novembre 2012), éd. par J. Jouanna, M. Zink, Paris 2014, pp. 217-229.

2016. (con R.W. Daniel). Two Hellenistic Medical Papyri of the Ärztekammer Nordrhein (P.ÄkNo 1 and 2) (Pap.Colon. XXXVIII), Paderborn 2016.

2018. 'Tipologia testuale e linguaggio tecnico nelle ricette su papiri: tre casi di ricette conservate dai PSI', in Parlare la medicina: fra lingue e culture, nello spazio e nel tempo. Atti del Convegno Internazionale (Parma, 5-7 Settembre 2016), a c. di N. Reggiani, con F. Bertonazzi, Firenze 2018, pp. 113-127.

2019. 'From Prescription to Practice: The Evidence of Two Medical Papyri from Roman Egypt', in Greek Medical Papyri: Text, Context, Hypertext. Proceedings of the DIGMEDTEX International Conference (Parma, 2-4 November 2016), ed. by N. Reggiani, Berlin - Boston 2019, pp. 3-17.

\section{I papiri e la scienza medica antica. Un'introduzione}

1993. 'L'apporto dei papiri alla conoscenza della scienza medica antica', «ANRW», II 37.1, Berlin - New York 1993, pp. 458-562.

2004. (con A. Marcone). Medicina, medico e società nel mondo antico, Firenze 2004.

\section{Forme di trasmissione della dottrina e dei testi medici}

1984. 'L'apporto dei papiri alla conoscenza dei medici pregalenici', in Atti del XVII Congresso Internazionale di Papirologia (Napoli, 19-26 maggio 1983), vol. II, Napoli 1984, pp. 351-355.

1992. 'I papiri e la tradizione medievale nella ricettazione dei testi medici tardoantichi', in Tradizione e ecdotica dei testi medici tardoantichi e bizantini. Atti del Convegno Internazionale (Anacapri, 29-31 ottobre 1990), a c. di A. Garzya, Napoli 1992, pp. 13-27.

1994. 'Precisazioni sulla data di alcuni testi di medicina in forma di codice', in Proceedings of the 20th International Congress of Papyrologists (Copenhagen, 23-29 August 1992), ed. by A. Bülow-Jacobsen, Copenhagen 1994, pp. 410-413.

1999. 'Riflessi e applicazioni della terapia ippocratica nella testimonianza dei papiri', in Aspetti della terapia nel Corpus Hippocraticum. Atti del IXe Colloque International Hippocratique (Pisa, 25-29 settembre 1996), a c. di I. Garofalo, A. Lami, D. Manetti, A. Roselli, Firenze 1999, pp. 431-446.

2000. 'Codici papiracei di medicina con scolî e commento', in Le commentaire entre tradition et innovation. Actes du Colloque International de l'Institut des traditions textuelles (Paris et Villejuif, 22-25 septembre 1999), éd. par M.-O. Goulet-Cazé, Paris 2000, pp. 37-52.

2003. 'L'esegesi del libro tecnico: papiri di medicina con scoli e commenti', Papiri filosofici. Miscellanea di studi IV (Studi e Testi per il Corpus dei Papiri Filosofici Greci e Latini 11), Firenze 2003, pp. 9-29. 
2006. 'Il 'gergo' grafico ed espressivo della ricettazione medica antica', in Medicina e società nel mondo antico. Atti del Convegno (Udine, 4-5 ottobre 2005), a c. di A. Marcone, Firenze 2006, pp. 142-167.

2007. 'Teaching Medicine in Late Antiquity: Methods, Texts and Contexts', in Form and Content of Instruction in Anglo-Saxon England in the Light of Contemporary Manuscript Evidence. Papers presented at the International Conference (Udine, 6-8 April 2006), ed. by P. Lendinara, L. Lazzari, M.A. D’Aronco, Turnhout 2007, pp. 385-398.

\section{Storia delle malattie}

2012a. 'Considerazioni sulla 'peste antonina' in Egitto alla luce delle testimonianze papirologiche', in L'impatto della 'peste antonina', a c. di E. Lo Cascio, Bari 2012, pp. 15-28.

2012b. ' 'Segni' di malattia nelle lettere dei papiri', in Actes du 26e Congrès international de Papyrologie (Genève, 16-21 août 2010), éd. par P. Schubert, Genève 2012, pp. 37-44.

2017. 'Environmental Diseases According to Papyri from Egypt and Medical Thought', in Pollution and the Environment in Ancient Life and Thought. Proceedings of the Conference (Berlin, 16-17 October 2014), ed. by O.D. Cordovana, G.F. Chiai, Stuttgart 2017, pp. 163-175.

\section{Pratiche e tecniche di cura}

1981. (con M. Manfredi). 'Cenni sulla valutazione della componente psicologica nella normativa attinente il parto nella letteratura medica antica', in Atti del I Congresso Congiunto ItaloFranco-Spagnolo di Psicoprofilassi Ostetrica (Perugia, 28-31 maggio 1980), a c. di M. Gerli, G. Gilardi, E. Giannone, Perugia 1981, pp. 1-14.

2007. 'Prescription and Practice in Greek Medical Papyri from Egypt', in Zwischen Magie und Wissenschaft. Ärzte und Heilkunst in den Papyri aus Ägypten, hrsg. von H. Froschauer, C. Römer, Wien 2007, pp. 23-33.

2012. 'Gli strumenti perduti di Galeno', «La Torre di Babele» 8 (2012), pp. 239-247.

2015. 'Egypt and the Medicinal Use of Papyrus According to Soranus and Other Physicians', in The Frontiers of Ancient Science. Essays in Honor of Heinrich von Staden, ed. by B. Holmes, K.-D. Fischer, Berlin - New York 2015, pp. 1-18.

2016a. 'Crossing the Borders Between Egyptian and Greek Medical Practice', in Popular Medicine in Graeco-Roman Antiquity: Explorations, ed. by W.V. Harris, Leiden - Boston 2016, pp. 161-172.

2016b. 'Le borse terapeutiche a vapore nella medicina antica', in Medica-Papyrologica. Specimina di ricerca presentati al convegno "Parlare la medicina" (Parma, 5-7 settembre 2016), a c. di N. Reggiani, Parma 2016, pp. 9-13.

\section{$\underline{\text { Strutture assistenziali }}$}

2006. (con A. Marcone). 'Salute, malattia e 'prassi ospedaliera' nell'Egitto tardoantico', in Poveri ammalati e ammalati poveri. Dinamiche socio-economiche, trasformazioni culturali e misure assistenziali nell'Occidente romano in età tardoantica. Atti del Convegno di Studi (Palermo, 1315 ottobre 2005), a c. di R. Marino, C. Molè, A. Pinzone, M. Cassia, Catania 2006, pp. 24-31.

2013. 'Lavori per un ualetudinarium a Vindolanda. Nota a T.Vindol. II 155,6', «ZPE» 184 (2013), pp. 271-273.

\section{$\underline{\text { Recensioni }}$}

1981. Rec. a P. Galigani, Il De lapidum virtutibus di Michele Psello, Firenze 1980, «Prometheus» 8 (1981), pp. 285-288. 
1983. Rec. a Ippocrate, Epidemie. Libro sesto, a c. di D. Manetti, A. Roselli, Firenze 1982, «RFIC» (1983), pp. 339-342.

1995. Rec. a M.-H. Marganne, L'ophthalmologie dans l'Égypte gréco-romaine d'après les papyrus littéraires grecs, Leiden - New York - Köln 1994, «CE» 70 (1995), pp. 310-315.

1999. Rec. a Ps. Alessandro di Afrodisia, Trattato sulla febbre, a c. di P. Tassinari, Alessandria 1994, «Athenaeum» 87 (1999), pp. 623-624.

2000. Rec. a Michigan Papyri XVII. The Michigan Medical Codex (ASP 35), ed. by L.C. Youtie, Atlanta 1996, «BO» 57 (2000), pp. 613-616.

$\underline{\text { Riedizioni complessive degli scritti di Isabella Andorlini sulla Papirologia medica }}$

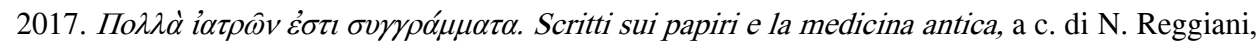
Firenze 2017.

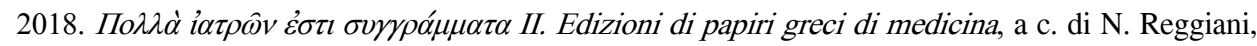
Firenze 2018.

\section{Edizioni di papiri letterari}

\section{Epica arcaica}

1997. 'PLB XXV 6 + P.Mon. II 35: Omero, Iliade A 384; 415-420', «ZPE» 115 (1997), pp. 197-198. 2000a. 'Hom. A 409-413', in Papiri dell'Iliade, a c. di M. Manfredi, Firenze 2000, p. 15 (n. 6). 2000b. 'Hom. H 441-448; 452-478', ibidem, pp. 47-51 (n. 18).

2000c. (con J. Lundon). 'Frammenti di Omero, Odissea XI 210-29 (PDuk inv. 60 + PPisaLit 23)', «ZPE» 133 (2000), pp. 1-6.

2001. 'Frammenti di Omero, Iliade nei papiri Aberdeen 145, 146a, 146', «Comunicazioni dell'Istituto Papirologico 'G. Vitelli'» 4 (2001), pp. 39-44.

\section{$\underline{\text { Oratoria }}$}

1994. 'Aeschines In Tim. 43-52', in The Oxyrhynchus Papyri, vol. LX, London 1994, pp. 67-74 (nr. 4030).

2001. (con R. Luiselli). 'Una ripresa di Diotogene Pitagorico, Sulla regalità, in PBingen 3 (encomio per Augusto?)', «ZPE» 136 (2001), pp. 155-166.

2003. 'Un nuovo frammento dell'Elena di Isocrate', in Studi sulla tradizione del testo di Isocrate (Studi e Testi per il Corpus dei Papiri Filosofici Greci e Latini 12), Firenze 2003, pp. 3-6.

2008. (con M. Fassino). 'Isocrates, Helena 11', in Corpus dei Papiri Filosofici Greci e Latini, vol. I.2*, cit., pp. 642-643.

2015. 'Aesch., In Ctes. 8; 41-43 (addenda a P.Oxy. LX 4041 = MP3 0010.01; LDAB 50)', in Charisterion per Revel A. Coles. Trenta testi letterari e documentari dall'Egitto (P. Coles), a c. di G. Bastianini, N. Gonis, S. Russo, Firenze 2015, pp. 31-37 (nr. 8).

\section{$\underline{\text { Prosa filosofica }}$}

1989a. 'Anaxilaus', in Corpus dei Papiri Filosofici Greci e Latini, vol. I.1*, Firenze 1989, pp. 170-171.

1989b. (con A. Linguiti). 'Aeschines Socraticus', ibidem, pp. 146-147.

1989c. (con A. Linguiti). 'Aristoteles HA IX 40, 624a34 (?)', ibidem, pp. 337-338.

1989d. (con A. Linguiti). 'Aristoteles Ath. 54, 2', ibidem, pp. 365-366. 
1989e. (con A. Linguiti e M. Manfredi). 'Elenco di libri', ibidem, pp. 99-105.

1992a. (con R. Laurenti) 'Musonius Rufus, An liberi omnes nati sint educandi', in Corpus dei

Papiri Filosofici Greci e Latini, vol. I.1**, Firenze 1992, pp. 480-490.

1992b. 'Musonius Rufus (PTura III 197, 27-200, 1-3)', ibidem, pp. 490-491.

1996. 'Un nuovo papiro di Plutarco (PSI inv. 2055: "Quaest. conv." IV)', in OAOI $\triangle I Z H \Sigma I O \Sigma$. Le

vie della ricerca. Studi in onore di Francesco Adorno, a c. di M.S. Funghi, Firenze 1996, pp. 3-10.

1999a. (con A. Linguiti). 'Pythagoras (PBrLibr Add Ms 37516, 1)', in Corpus dei Papiri Filosofici

Greci e Latini, vol. I.1***, Firenze 1999, pp. 681-684.

1999b. (con A. Linguiti). 'Pythagorei (PTura III 79, 26-80, 1)', ibidem, pp. 686-688.

2000. 'Platone, Fedone, 60d-e', in Papyri in honorem Johannis Bingen octogenarii, cur. H. Melaerts, Leuven 2000, pp. 47-51 (nr. 10).

2016. 'Frammento filosofico', in E sì d'amici pieno. Omaggio di studiosi italiani a Guido Bastianini per il suo settantesimo compleanno, a c. di A. Casanova, G. Messeri, R. Pintaudi, vol. I, Firenze 2016, pp. 39-43 (nr. 6).

\section{Edizioni di papiri documentari}

1982. (con G. Sodini). 'Loan of Wheat', in The Oxyrhynchus Papyri, vol. XLIX, London 1982, pp. 201-204 (nr. 3493).

1983a. 'Registrazione di terreni e lista di colori', in Trenta testi greci da papiri letterari e documentari editi in occasione del XVII Congresso Internazionale di Papirologia, cit., pp. 60-64 (nr. 18).

1983b. 'Nomina liturgica', ibidem, pp. 104-108 (nr. 28).

1983c. 'Inizio di richiesta di registrazione fiscale', ibidem, pp. 109-112 (nr. 29).

1985a. 'Lettera ufficiale', in The Rendel Harris Papyri of Woodbrooke College, Birmingham, vol.

II, Zutphen 1985, pp. 96-100 (nr. 203).

1985b. 'Transazione conseguente una disputa di proprietà', ibidem, pp. 153-161 (nr. 228).

1995a. 'Frammento di registro. Notizia di una decisione prefettizia', in Dai Papiri della Società

Italiana. Omaggio al XXI Congresso Internazionale di Papirologia, cit., pp. $96-99$ (nr. 16).

1995b. 'Verbale di processo', ibidem, pp. 100-104 (nr. 17).

1999. 'Riedizione di PSI XIII 1311: contratto di mutuo in denaro', «AnPap»10-11 (1998-1999), pp. 119-129.

2001. 'Ricevuta di un versamento in grano al thesauros di Somolò', in Studium atque urbanitas. Miscellanea in onore di Sergio Daris («PapLup»9), a c. di M. Capasso, S. Pernigotti, Galatina 2001, pp. 9-14.

2006. (con K. Maresch). Das Archiv des Aurelius Ammon, vol. II, A+B (Pap.Colon. XXVI/2), Paderborn - München - Wien - Zürich 2006.

2007. 'Disposizioni agricole per Theon', in Papyri in Memory of P.J. Sijpesteijn, ed. by A.J.B. Sirks, K.A. Worp, Oakville (CT) 2007, pp. 355-362 (nr. 56).

2008. 'Old and New Greek Papyri from Tebtunis in the Bancroft Library of Berkeley: Work in Progress', in Graeco-Roman Fayum - Texts and Archaeology, cit., pp. 9-13.

2015a. 'Women's Letters Concerning Textile Manufacture (P.Tebt. II 413-414)', in Von der Pharaonenzeit bis zur Spätantik. Kulturelle Vielfalt im Fayum. Akten der 5. Internationalen FayumKonferenz, (Leipzig, 29. Mai bis 1. Juni 2013), hrsg. von N. Quenouille, Wiesbaden 2015, pp. 1-16.

2015b. (con K. Maresch). 'Ein neues Bruchstück aus dem Ammon-Archiv. Entwurf eines Vertrags über den Verkauf von Sklaven des Harpokration (P.Ammon II 48 erweitert)', «ZPE» 193 (2015), pp. 231-240.

2018. 'SB XXIV 16147 (P.Vindob. G 31787): lista alimentare', in offerti a Mario Capasso, a c. di P. Davoli, N. Pellé, Lecce 2017, pp. 1-4. 


\section{Storia sociale, economica e culturale dell'Egitto antico}

1998. 'I colori dei tessuti', in Antinoe cent'anni dopo. Catalogo della mostra (Firenze, Palazzo Medici-Ricciardi, 10 luglio-1 novembre 1998), a c. di L. Del Francia Barocas, Parigi - Firenze 1998, pp. 154-160.

1999a. (con A. Marcone). 'L'orzo nell'Egitto greco-romano', in Demografia, sistemi agrari, regimi alimentari nel mondo antico. Atti del Convegno Internazionale di Studi (Parma, 17-19 ottobre 1997), a c. di D. Vera, Bari 1999, pp. 325-344.

1999b. Rec. a J. Bingen, A. Bülow-Jacobsen, W.E.H. Cockle, H. Cuvigny, F. Kayser, W. Van Rengen, Mons Claudianus. Ostraca graeca et latina II. O.Claud. 191 à 416, Le Caire 1997, «AnPap» 10-11 (1998-1999), pp. 257-260.

1999c. Rec. a D.P.S. Peacock, V.A. Maxfield, Survey and Excavation. Mons Claudianus. 19871993, Volume I: Topography \& Quarries, Le Caire 1997, ibidem, pp. 260-261.

2000. 'Cronaca del XXII Congresso Internazionale di Papirologia (Firenze, 23-29 agosto 1998): il contributo alla storia della tradizione cristiana in Egitto', «Adamantius» 6 (2000), pp. 393-398.

2007. (con K. Maresch). 'Aus der Arbeit am Ammon-Archiv: Landkulturen und Landparzellen in einer Besitzdeklaration aus Panopolis', in Von Noricum nach Ägypten: Eine Reise durch die Welt der Antike. Aktuelle Forschungen zu Kultur, Alltag und Recht in der römischen Welt. Beiträge der Tagung „Noricum in vorrömischer und römischer Zeit: Forschungsstand und Neuansätze (Klagenfurt, 3-4.10.2003)“ und des „Klagenfurter Papyrologentages (29.30.10.2003) “, hrsg. von K. Strobel, R. Lafer, Klagenfurt - Ljubljana - Wien 2007, pp. 221-236.

2010. 'Paganesimo e Cristianesimo nell'Egitto del IV secolo d.C.: le carte di Ammon, scholasticus di Panopoli', «Anabases» 12 (2010), pp. 13-21.

2015. 'Il pane nell'Egitto romano', in La civiltà del pane. Storia, tecniche e simboli dal Mediterraneo all'Atlantico. Atti del Convegno Internazionale di Studi (Brescia, 1-6 dicembre 2014), a c. di G. Archetti, vol. I, Spoleto 2015, pp. 461-468.

2016. 'Import of Luxury Goods in the Light of the Papyri of the Roman Period', in Proceedings of the 27th International Congress of Papyrology (Warsaw, 29 July-3 August 2013), ed. by T. Derda, A. Lajtar, J. Urbanik, vol. III, Warsaw 2016, pp. 1927-1941.

2018a. Voci 'Ammon Scholasticus'; 'Panopolis'; 'Panopolis Papyri’; 'Writing, Greek and Coptic', in The Oxford Dictionary of Late Antiquity, ed. by O. Nicholson, Oxford 2018.

2018b. 'Papiri e scritture nella collezione egizia del Museo Archeologico Nazionale di Parma', in Papiri, medicina antica e cultura materiale. Contributi in ricordo di Isabella Andorlini, a c. di N. Reggiani e A. Bovo, Parma 2018, pp. 17-22.

\section{Storia della Papirologia}

1995. 'Scavi e acquisti di papiri negli anni '30: il caso dei PLund', «Comunicazioni dell'Istituto Papirologico 'G. Vitelli'» [1] (1995), Firenze 1995, pp. 45-50.

1998. 'Gli scavi di John de Monins Johnson ad Antinoe (1913-1914)', Antinoe cent'anni dopo, cit., pp. 19-22.

2003. (con G. Bastianini, M. Manfredi, G. Menci). L'Istituto Papirologico “G. Vitelli”. Storia, scavi e collezioni / History, Excavations and Collections, Firenze 2003 (CD-ROM).

2004. 'La collezione dei papiri demotici dell'Istituto Papirologico "Girolamo Vitelli" a Firenze', in Res severa verum gaudium. Festschrift für Karl-Theodor Zauzich zum 65. Geburtstag am 8. Juni 2004, hrsg. von F. Hoffmann, H.J. Thissen, Leuven - Paris - Sterling 2004, pp. 13-26.

2009. 'Papiri e papirologia a Parma', «A\&R» n.s. II, 3/3-4 (2009), pp. 229-233. 


\section{Tecnologie digitali per la ricerca papirologica}

1993. (con G. Menci, D. Bertani, M. Cetica, P. Poggi). 'Use of CCD Cameras and Red-Extended Photographic Film for Inspection and Recording of Dark Papyri', «Science and Technology for Cultural Heritage» 2 (1993), pp. 115-122.

2001. (con F. Lucarelli, P.A. Mandò). 'Particle-Induced X Ray-Emission for the Analysis of Writing and Painting Materials on Papyri and Textiles from Graeco-Roman Egypt', in Atti del XXII Congresso Internazionale Di Papirologia (Firenze, 23-29 Agosto 1998), a c. di I. Andorlini, G. Bastianini, M. Manfredi, G. Menci, vol. I, Firenze 2001, pp. 51-64.

2007. (con A. Agnesini, M. Magnani, S. Santoro, A.M. Tammaro). 'Tecnologie digitali e ricomposizione dell'antico: papiri, siti archeologici, biblioteche ed archivi digitali', «Griseldaonline» 6 (2006-2007), http://www.griseldaonline.it/informatica/tecnologie-digitali-ericomposizioni-dell-antico.html.

2008. 'Ricongiungere virtualmente archivi papiracei dispersi: le carte di Ammon, advocatus', in Scienze umane e cultura digitale, a c. di A.M. Tammaro, S. Santoro, Fiesole 2008, pp. 167-176.

2012. (con N. Reggiani). 'Edizione e ricostruzione digitale dei testi papiracei', in Diritto romano e scienze antichistiche nell'era digitale. Convegno di studio (Firenze, 12-13 settembre 2011), a c. di N. Palazzolo, Torino 2012, pp. 131- 146.

\section{Curatela di Congresso Internazionale di Papirologia}

2001. (a c. di, con G. Bastianini, M. Manfredi, G. Menci). Atti del XXII Congresso Internazionale di Papirologia (Firenze, 23-29 agosto 1998), voll. I-II, Firenze 2001.

\section{Contributi in memoria di Isabella Andorlini}

2017a. N. Reggiani, 'Isabella Andorlini (28 aprile 1955 - 11 novembre 2016)', «Aegyptus» 96 (2016) [2017], pp. 143-165.

2017b. 'A Isabella Andorlini', «Galenos» 10 (2016) [2017], p. 9.

2018. Papiri, medicina antica e cultura materiale. Contributi in ricordo di Isabella Andorlini, a c. di N. Reggiani e A. Bovo, Parma 2018 [A. Jördens, 'In Erinnerung an Isabella Andorlini'; A. Roselli, 'Ricordo di Isabella Andorlini'; G. Bastianini, 'Isabella Andorlini all' "Istituto Vitelli" di Firenze'; U. Fantasia, 'Isabella Andorlini docente di Papirologia a Parma'; G. Azzarello, 'Isabella Andorlini e la Papirologia letteraria'; F. Reiter, 'Isabella Andorlini e la Papirologia documentaria' R. Conversi, 'Isabella Andorlini e i papiri di Parma'; M. Botti, 'Isabella Andorlini e Giuseppe Botti'; N. Reggiani, 'Isabella Andorlini e la Papirologia medica']

2019. N. Reggiani, 'Isabella Andorlini (1955-2016)', in Hermae. Scholars and Scholarship in Papyrology, V, ed. by M. Capasso, Pisa - Roma 2018 (in c.d.s.). 



\section{INDICES}


